International Journal of Research in Nursing 1 (1): 21-24, 2010

ISSN 1949-0194

(C) 2010 Science Publications

\title{
A Study of Clinical Behavior in Intensive Care Unit
}

\author{
Gilly Smith \\ School of Nursing, Midwifery and Post Graduate Medicine, \\ Edith Cowan University, 100 Joondalup Drive, Joondalup, Western Australia, 6027
}

\begin{abstract}
Problem statement: In order to support the needs of the staff working in discrete areas of nursing, the attitudes, behaviors and needs of the group must first be articulated. Unfortunately, there has been little research conducted to understand the culture of what it is that health care workers think and feel and why they make the choices they do in their working environment and also why they chose to leave the environment. Specifically, what do intensive care clinicians think about the work they do when managing end-of-life care in this technical, highly skilled environment. The focus of an ethnographic study conducted in an Australian hospital in 2007 was to describe the attitudes, behaviors and social conscience of this group whilst they provided end-of-life care. Conclusion: Staff were unable to control many factors that impact on the capacity of professional practice within the intensive care environment. In response to this limited control, staff developed tactics for managing the varying levels of expected clinical service delivery associated with varying possible outcome for each individual patient admission. This research garnered three discrete groups of coping strategies: physical, social and psychological. Future research may demonstrate capacity to engage in such coping strategies has a direct link to clinical longevity.
\end{abstract}

Key words: End-of-life care, intensive care unit, clinician behavior

\section{INTRODUCTION}

This study describes the behaviors of Intensive Care Unit (ICU) clinicians observed during a twelve month participant observation ethnographic study. Human resources are responsible for $70-80 \%$ of the Australian health care budget and are the single greatest cost within intensive care settings. There has been little research conducted to understand the culture of what it is that health care workers think and feel and why they make the choices they do in their working environment (Turnbull et al., 2005). The focus of this ethnographic study was to articulate the attitudes, behaviors and social conscience of this group whilst they provided end-of-life care as opposed to any gendered research deliberation. The research findings indicate there were several themes observable within the attitudes and behaviors of the study group.

The changing role of ICU: Appropriate health care is when the expected health benefits exceed the expected negative consequences by a wide enough margin to justify treatment (NSW Department of Health, 2005). Inappropriate health care is therefore when patients receive care that contributes to over or under use of health care services. Intensive care has developed so rapidly that associated ethical and economic considerations of appropriate use of the service have not kept in step with the technological advances (Levy and McBride, 2006).

At the same time the exposure the general populous have to media images of intensive care potential has contributed to an increase for intensive care services from a social demand rather than clinically based need (Lanken et al., 2008). For patients this has resulted in a transition from rapid deaths because of hypoxia or hypotension, to prolonged deaths that result from sepsis or multiple organ failure. For clinicians this has resulted in an increasing volume of patients requiring end-of-life management in the intensive care setting (Harrison et al., 2007). And the capacity of individual ICU clinicians to manage the increasing demand for ICU services for end-of-life care was most affected by factors arising from outside the ICU (Luce and Rubenfeld, 2002; Nyman and Sprung, 2000).

Primary amongst these was the limited control over ICU admission. Nominally the intensivist on duty and in their absence the senior ICU registrar, had final say in which patients were admitted to ICU. The observed reality was that decisions to perform surgery were made without ICU consultation and patients required ICU post-operatively. While many of these patients were booked admissions, ICU was not advised of their pre- 
operative status and on occasion patients with multiple co-morbidities arrived at the ICU after simple procedures with very poor prognosis. The issues of external factors impacting on ICU management raised in this study have been reviewed by other research (Prendergast and Puntillo, 2002; Rocker et al., 2004; Poncet and Toullic, 2007), but not how the staff manage these factors. Other surgeries were performed without ICU beds being booked, yet the patients required ICU management post-operatively. It was acknowledged by staff there was going to be a percentage of patients for whom this need could not be predicted. Conversely, there was a larger percentage of non-booked post-operative admissions that staff determined it was either futile surgery, last chance surgery with a limited chance of survival, or low risk surgery on high anaesthetic risk patients. The sentiment was amongst ICU nursing staff, had ICU staff been asked to review these patients pre-operatively, surgery would not have been undertaken. They felt ICU was left to manage the patient and their family through the dying process unnecessarily.

This sentiment was repeated with patients who had sustained out of hospital cardiac or respiratory arrests. Often these patients would be brought in by ambulance effectively moribund, without any chance of recovering to their pre-arrest status. The demands of society have resulted in an expectation placed on Emergency Department physicians to offer ICU services. This has resulted (anecdotally from narratives in this study) in an increasing number of patients being transferred to ICU for compassionate ventilation whilst the family comes to terms with the poor prognosis. The impact on ICU clinicians is the requirement to manage the family in their grief, instead of this being managed by the staff in the Emergency Department. ICU staff additionally indicated their belief this practice was detrimental to the families as it offered false hope of recovery where as the previous practice of declaring them unrecoverable in the Emergency Department gave the families an accurate assessment of the prognosis and provided them the opportunity to plan for the impending death.

A third source of uncontrollable admission of patients to ICU were physicians who failed to develop a management plan for end-of-life care with the patients at a time that they were well enough to indicate their own wishes (Rescher, 1969). These patients would deteriorate on medical wards to such a point that the intra-hospital emergency call would be activated. This resulted in staff from the ICU having to review and take over management of these patients in the absence of any clear directive from either the patient or the physician managing their care. Once again, this lack of clearly articulated management plan resulted in ICU staff being required to manage medical patients at the end of their life, inclusive in this process was implementing appropriate end-of-life directives in conjunction with the patient's family. The sentiment of staff in these situations was that ICU were left to clean up the unfinished work of medical staff.

Staff were unable to control many factors that impacted on the capacity of professional practice within this environment. In response to this limited control, staff were noted to develop tactics for managing the varying levels of expected clinical service delivery associated with varying possible outcome for each individual patient admission. This research garnered three discrete groups of coping strategies: physical, social and psychological.

Physical strategies: The clinicians under observation used the physical structure of the intensive care to limit access to their working environment. This was achieved by limiting the number of visitors permitted at the bedside. Visitors required staff to admit them to the unit and at times this limit on access was a strategy used to allow time for staff to interact with one another or to interact with patients without visitors present.

Staff were observed to use physical behaviors and gestures to manage their interactions with one another, with clinicians from external to the unit and with patient visitors. This included ensuring immaculate physical presentation to reduce intimidation by individuals who attempted to use their physical or social power to intimidate (especially nursing) staff; use of medical jargon by junior staff to cover personal discomfort when discussing prognosis with family members and focusing on task-oriented work.

Some staff requested assignment to particular patient-types to avoid provision of end-of-life care. When assigned to care for patients at the end of their life, some clinicians were observed to limit the time they spent with the patient and family to the minimum necessary for primary nursing cares to be provided. They were observed to physically remove themselves from the bed area and find other clinical tasks to fill their shift.

Social strategies: The intensive care clinicians observed throughout this study used a combination of socializing with their work colleagues and strategies to manage the work they do outside of the working environment. Social gatherings where ICU clinicians were present often degenerated into tales of gore and graphic descriptions of their work. These gatherings were observed most often after particularly busy or 
challenging shifts. Often staff from the Emergency Department, police or ambulance service participated in these impromptu debriefing sessions.

The families of ICU clinicians also reported occasions where the clinical work spilled into the dinner conversation, family barbeque and even discussions about food preparation. The children of ICU clinicians were observed to be quite clear about the work their parents performed and at times adopted the same capacity for sharing graphic clinical details with one another. There was an acceptance by the families this was a normal component of living with an intensive care clinician and reported failure by people outside of their cohort to accept this as being a deficit of the outsider's character.

There was debate amongst staff about the value of formalized religions in supporting individuals working in ICU. However there was little argument each clinician required a measure of faith that there was some higher purpose to their work. The staff were divided into the groups who sought solace and support from their religious affiliations and those who did not. Despite this dichotomy, the two groups respected each other's choices and at times supported their need to demonstrate their religious beliefs (prayer at the bedside with families).

Psychological strategies: Clinicians within the study described a process of mental risk assessment conducted by individual clinicians as a self-preparatory risk evaluation for each patient. This risk assessment examined how significant the impact on their individual workload. In the situation of the team leader, what impact each admission may have on the capacity of the unit as a whole, to provide the level of service appropriate to the changing levels of clinical need. The accuracy of this unacknowledged 'mental triage' tool appeared to be no less than formalized risk assessment tools. 'Mental triage' allowed individual clinicians to separate in their own mind which patients were likely to survive and had a greater likelihood of death. Assigning patients to survivability groupings involved in this process allowed clinicians to prepare mentally for the impending death and the psychosocial impact of providing end-of-life care.

\section{CONCLUSION}

The clinicians described their frustration with having to be "all things to all people" in the absence of their out-of-ICU colleagues fulfilling the responsibilities of the roles they took on within the hospital setting. The ICU clinician sees the purpose of ICU as providing support to patients during a period of physical crisis that cannot be managed outside of the highly technical and invasive ICU environment. Bluntly, ICU is about caring for the patients who can be salvaged.

The people working within this setting were observed to be a unique group, having an imaginary demarcation line separating them from the wider community in response to their exposure to the most basic of human frailties-death. Unlike other medical specialties that accept death a natural component of disease process (such as cancer care) ICU clinicians spent a vast majority of their time trying to prevent death. The move toward admitting patients for end-oflife care in response to poorly documented management plans on medical wards; surgical intervention for frail patients or those with multiple co-morbidities or high anaesthetic risks or out of hospital arrests does not fit within the concept of "caring for the salvageable patient". This has led to role ambiguity for ICU nurses. One moment they are using all their skills and knowledge to save the young car accident victim and in the next breath they are required to ensure the passing of an elderly patient with end-stage chronic airways limitation is pain free and dignified as possible.

As a result of this role ambiguity, or changing role, clinicians were observed to have developed three observable types of coping strategies: physical, social and psychological. Whether these observable behaviors were unique to the study setting or are transferable observations to be seen in other ICUs, is for future research to discover.

\section{REFERENCES}

Harrison, D.A., G.J. Parry, J.R. Carpenter, A. Short and K. Rowan. 2007. A new risk prediction model for critical care: The Intensive Care National Audit and Research Centre (ICNARC) model. Crit. Care Med., 35: 1091-1098. DOI: 10.1097/01.CCM.0000259468.24532.44

Lanken, P.N., P.B. Terry, H.M. DeLisser, B.F. Fahy, J. Hansen-Flaschen et al., 2008. An official American thoracic society clinical policy statement: Palliative care for patients with respiratory diseases and critical illnesses. Am. J. Respir. Crit. Care Med., 177: 912-927. DOI: $10.1164 / \mathrm{rccm} .200605-587 \mathrm{ST}$

Levy, M.M. and D.L. McBride, 2006. End-of-life care in the intensive care unit: State of the art in 2006. C. Care Med., 34: S306-308. DOI: 10.1097/01.CCM.0000233788.96388.D8 
Luce, J.M. and G.D. Rubenfeld, 2002. Can health care costs be reduced by limiting intensive care at the end of life? Am. J. Respir. C. Care Med., 165: 750-754. DOI: $10.1164 / \mathrm{rccm} .2109045$

NSW Department of Health, 2005. Guidelines for Endof-Life Decision Making. New South Wales Department of Health, ISBN: 0734737858.

Nyman, D.J. and C.L. Sprung, 2000. End-of-life decision making in the intensive care unit. Intens. Care Med., 26: 1414-1420. DOI: 10.1007/s001340000641

Poncet, M.C. and P. Toullic, 2007. Burnout syndrome in critical care nursing staff. Am. J. Respir. C. Care Med., 175: 698-704. DOI: 10.1164/rccm.200606$806 \mathrm{OC}$
Prendergast, T.J. and K.A. Puntillo, 2002. Withdrawal of Life Support; Intensive caring at the end of life. J. Am. Med. Assoc., 288: 2732-2740. DOI: 10.1164/rccm.200606-806OC

Rescher, N., 1969. The allocation of exotic medical lifesaving therapy. Ethics, 79: 173-186. http://www.jstor.org/stable/2379841

Rocker, G., D. Cook, P. Sjokvist, B. Weaver and S. Finfer et al., 2004. Clinician predictions of intensive care unit mortality. C. Care Med., 323: 1231-1232. PMID: 15190965

Turnbull, E., A. Flabouris and R. Iedema, 2005. An outside perspective of the life-world of ICU. J. C. Care, 18: 71-75. DOI: 10.1016/S10367314(05)80005-0 